\title{
Improvement of ICRF antenna loading by local gas injection on ASDEX Upgrade
}

\author{
P. Jacquet ${ }^{1}$, V. Bobkov ${ }^{2}$, M.-L. Mayoral ${ }^{1}$, I. Monakhov ${ }^{1}$, J. M. Noterdaeme ${ }^{2,3}$, A. Scarabosio ${ }^{2}$, \\ I. Stepanov ${ }^{2}$, M. Vrancken ${ }^{4}$, E. Wolfrum ${ }^{2}$ and ASDEX Upgrade Team. \\ ${ }^{1}$ EURATOM/CCFE Fusion Association, Culham Science Centre, Abingdon, OX14 3DB, UK. \\ ${ }^{2}$ Max-Planck-Institut für Plasmaphysik, EURATOM-Association IPP, D-85748 Garching, \\ Germany. \\ ${ }^{3}$ Gent University, EESA Department, Gent, Belgium. \\ ${ }^{4}$ LPP/ERM-KMS, Association EURATOM-Belgian State, Brussels, Belgium.
}

E-mail address of the main author: philippe.jacquet@ccfe.ac.uk

\begin{abstract}
Local gas injection has recently been tested as a tool to improve coupling of Ion Cyclotron Range of Frequency (ICRF) waves to tokamak plasmas, which relies on the tunneling of the fast wave between the antenna structure and the cut-off layer within the plasma. It is found that antenna loading resistance is substantially increased when using a local gas inlet at a deuterium gas injection rate of $5 \times 10^{21} \mathrm{el} / \mathrm{s}$ or higher, and that the proximity of the gas inlet to the antenna is the key factor to optimize the improvement in coupling due to gas injection.
\end{abstract}

PACS numbers: 52.55 .Fa, 52.50.Qt

Ion Cyclotron Range of Frequency (ICRF) wave heating of tokamak plasmas relies on the propagation of the fast wave, which is evanescent until it reaches a plasma density that exceeds the cut-off value (typically in the $10^{18} \mathrm{~m}^{-3}$ range). Given $V_{\max }$, the maximum operating RF voltage in the antenna structure (or in the ICRF system transmission lines) limited by voltage stand-off, the achievable coupled power varies to a first approximation linearly with the antenna resistance $R_{c}: P_{\text {coupled }} \sim V_{\max }^{2} R_{c} / 2 Z_{c}^{2}$, where $Z_{c}$ is the transmission line characteristic impedance. $R_{c}$ accounts for the power transferred to the fast wave or other plasma related wave propagation or RF absorption mechanisms. Typically, $R_{c}$ decreases exponentially with the distance between the antenna and the cut-off density layer [1] [2], hence the coupling properties of the antenna depend critically on the density profile in the Scrape-Off Layer (SOL) at the edge of the plasma. In ITER there are significant uncertainties in the density profiles predicted for the SOL, which lead to large uncertainties in the coupled power obtained from antenna simulation codes [3]. Recently it was proposed to use local gas injection as a tool to minimise the sensitivity of the ICRF coupling to the edge density and thus reduce the uncertainty in the prediction of the coupled power. Experiments to assess the 
efficiency of this tool have been performed on several tokamaks [4], including JET [5], ToreSupra, TEXTOR, and DIII-D [6]. This letter highlights results of the experiments carried out on ASDEX Upgrade (AUG) aiming at the characterisation of the effect of local gas injection on ICRF wave coupling. In particular, the goal of the experiment was to study the loading resistance of each antenna, as a function of gas inlet location, gas injection level, plasmaantenna distance and ICRF power level. It was also considered important to determine whether the loading resistance changes due to gas injection could be explained by changes in the electron density in the SOL.

AUG is a divertor tokamak with a full-tungsten first wall with $R_{\text {maj }} \sim 1.65 \mathrm{~m}, R_{\min } \sim$ $0.5 \mathrm{~m}, \mathrm{~B}_{\mathrm{T}}<3.1 \mathrm{~T}, \mathrm{I}_{\mathrm{p}}<1.6 \mathrm{MA}$ and pulse duration $<10 \mathrm{sec}$. Figure 1 is a top view of the tokamak showing the four ICRF antennas used during the experiment. Each antenna extends over 0.86 $\mathrm{m}$ vertically, and each comprises 2 straps which are normally configured with a $\pi$ toroidal phase difference (i.e. dipole configuration). The antennas radiate a fast wave with a maximum spectral power at $\mathrm{k}_{/ /, 0} \sim 8 \mathrm{~m}^{-1}$. The ICRF antenna system is sketched in Figure 2. Antennas are paired through a $3 \mathrm{~dB}$ hybrid coupler arrangement which provides more favourable matching conditions to the generators, especially during plasma perturbations such as ELMs. During the experiment antennas 1 and 3 were paired and, similarly, antennas 2 and 4. Pairing antennas that are located on opposite sides of the tokamak minimises the magnetic connection and eliminates cross-talk between the antennas in each pair due to SOL density changes caused by antenna operation, which propagate along field lines connected to the antenna [7]. RF matching of the transmission lines is performed using a double stub arrangement. RF measurements from directional couplers located before the stubs, and RF probes allow evaluation of the coupling resistance of each strap after subtraction of the vacuum losses (see the Appendix). Three gas inlets located in the machine mid-plane were used during the experiment, a local gas inlet embedded in one of the protection limiters of Antenna-4 referred as 'ICR-4', 'A1' and 'A13' (see Figure 1). The injected amount of gas from the inlets is controlled using calibrated piezo-valves, and typical time response of the system is $\sim 150 \mathrm{~ms}$. The location of the Li-beam diagnostic used to measure the electron density in the SOL is also shown in Figure 1. The Li-beam line of sight is located $\sim 0.3 \mathrm{~m}$ above the AUG equatorial plane.

The experiments described here were conducted using Deuterium (D) plasmas with Hydrogen $(\mathrm{H})$ minority ( 1 to $2 \% \mathrm{H}$ concentration in the plasma centre). ICRF wave heating at $\mathrm{P}_{\mathrm{ICRF}} \sim 1.2 \mathrm{MW}$ was the only auxiliary system used to heat the plasma. The confinement 
regime was L-mode, but some indications of ELMs were observed due to the close proximity to the H-mode power threshold. The ELM frequency was $\sim 100 \mathrm{~Hz}$ and some changes in the baseline level of the $\mathrm{D}_{\alpha}$ emission and central density were seen when the ICRF power level was increased. The magnetic field was $\mathrm{B}_{\mathrm{T}}=2 \mathrm{~T}$ and the ICRF frequency was $\mathrm{f}_{\mathrm{ICRF}}=30 \mathrm{MHz}$.

Figure 3 shows the time evolution for a typical pulse from this experiment. For good reproducibility all pulses were started with the injection of $\mathrm{D}_{2}$ gas from the A13 inlet. From $\mathrm{t}$ $=1.2 \mathrm{~s}$, two other gas inlets were successively used. In the example of pulse 25634 , ICR-4 was used between $1.2 \mathrm{~s}$ and $2.6 \mathrm{~s}$, and A1 was used between $2.6 \mathrm{~s}$ and $4 \mathrm{~s}$. The separatrix outer radius, $\mathrm{R}_{\text {out }}$, was moved away from the antennas during the $1^{\text {st }}$ gas phase, and back towards the antennas during the $2^{\text {nd }}$ gas phase. Throughout the pulse the antenna pair in use was switched alternately between antennas 2-4 and antennas 1-3. In this particular pulse, we could quantify the effect of gas injection from A1 and ICR-4 (9.5 el/s injection rate) on all four antennas for a separatrix-antenna distance ranging between $4.5 \mathrm{~cm}$ and $10.5 \mathrm{~cm}$. For example the effect from switching from ICR-4 (local to Antenna 4) to A1 results in a decrease in $\mathrm{R}_{\mathrm{c}} 4$ (coupling resistance of antenna 4 ) at $\mathrm{t} \sim 2.6 \mathrm{~s}$ and correspondingly a decrease in the maximum voltage in the transmission lines feeding the straps of antenna 4 (not shown). Reversely, $R_{c} 1$ improves (comparing values at $t \sim 3.1 \mathrm{~s}$ vs $\mathrm{t} \sim 2.3 \mathrm{~s}$ ) when switching to valve A1. However, no real-time ICRF power control was used to compensate the changes in antenna loading during this experiment. Also the matching stubs position was fixed during the pulses, and best RF power matching was achieved for low antenna loading, this condition also corresponds to higher net ICRF power transferred to the plasma via the antennas. This explains the counter-intuitive increase of RF power in the phases when the loading resistance decreases in Figure 3.

In Figure 4 the coupling resistance of antennas 1 and 4 is plotted as a function of the separatrix-antenna distance for different gas inlets and amounts of injected gas. Each antenna has a specific response, for example antenna 4 exhibits higher coupling resistance, and it is more susceptible to changes in the plasma-antenna distance and changes in the gas inlet and gas dosing. However, the following general features can be observed for all antennas:

- The coupling resistance decreases with increasing the plasma-antenna distance, consistent with the fact that the cut-off layer is further from the antenna and in line with previous ICRF antenna coupling characterisation experiments [1].

- The coupling resistance increases with the level of injected gas. 
- For a given gas injection rate, using gas inlets located close to the antenna enhances the coupling further. For antenna 4, coupling is improved most significantly when using ICR-4, and similarly antenna 1 coupling benefits most from the use of A1.

The importance of the gas inlet location for all four antennas is also illustrated in Figure 5. We define the coupling resistance increase rate due to gas injection for each antenna as $\kappa=\Delta R_{c} /\left(R_{c_{-} \text {reference }} \times \Delta \Gamma\right)$, where $\Delta R_{c}=R_{c^{-}} R_{c_{-} \text {reference }}$ is the increase of coupling resistance when increasing the deuterium gas injection rate $\Gamma$, by $\Delta \Gamma=\Gamma-\Gamma_{\text {reference. }} R_{c_{-} \text {reference }}$ and $\Gamma_{\text {reference }}$ correspond to distant mid-plane fuelling at small flow rate $\left(\sim 5 \times 10^{21} \mathrm{el} / \mathrm{s}\right)$. For the four antennas and three gas inlets used, $\kappa$ calculated for flow rate $\Gamma \sim 9.5 \times 10^{21} \mathrm{el} / \mathrm{s}$ is plotted as a function of $\rho$ representing the antenna-gas inlet distance defined as $\rho=1 / \alpha$ with $\alpha=\frac{1}{\left|\Phi_{\text {ant }}-\Phi_{\text {inlet }}\right|_{\text {clockwise }}}+\frac{1}{\left|\Phi_{\text {ant }}-\Phi_{\text {inlet }}\right|_{\text {counter-clockwise }}} . \Phi$ is the toroidal angle (in radians) of the location of the gas inlets or of the antennas. The clockwise and counter clockwise paths between the inlets and the antennas are taken into account in the definition of $\rho$. From Figure 5 we can define a zone corresponding to $\rho>1$ (i.e. gas inlets far away from the antennas) for which the coupling resistance increase rate is relatively insensitive to the distance between the antenna and the inlet. This zone is representative of the coupling resistance improvement from a global electron density increase in the SOL due to gas injection. For $\rho<1$ (i.e. gas inlets close to the antennas) the coupling improves significantly as the inlet-antenna distance decreases, all antennas showing the same trend. This corresponds to a local effect of gas injection on antenna coupling.

We shall now relate antenna coupling improvement to the electron density increase in the SOL. Density profiles were obtained from a probabilistic analysis of the Li-beam diagnostic (SOL and edge) and interferometry (edge and plasma centre) measurements [8] [9]. Examples zooming in the plasma edge and SOL region with moderate $\left(5 \times 10^{21} \mathrm{el} / \mathrm{s}\right)$ or strong $\left(9.5 \times 10^{21} \mathrm{el} / \mathrm{s}\right)$ gas puffing are shown in Figure 6. In these examples the central density was not significantly changed by the increased gas injection rate; however a slight decrease of the density at the pedestal can be noticed at the higher gas injection rates. In the SOL, increasing the gas injection rate leads to an increase of the measured density, irrespective of which gas inlet is used. This can be expected to improve the antenna loading by reducing the distance between the antenna and the cut-off layer. In order to relate the antenna coupling 
enhancement observed with gas puffing to the SOL density measurements, we have proceeded as follows:

a) For each inlet used, the changes in the cut-off position were determined while the gas injection rate was increased from $5 \times 10^{21} \mathrm{~s}^{-1}$ to $9.5 \times 10^{21} \mathrm{~s}^{-1}$. These measurements were made in two conditions: (i) with antennas 1-3 powered; (ii) with antennas 2-4 powered.

b) The expected changes in coupling resistance from changes in density profiles were estimated. Although full antenna modelling using a code such as TOPICA [10] would be ideal for this purpose, a simplified approach was adopted based on an experimental characterisation of the dependence of the antenna loading on cut-off layer position. This approach is related to the analysis presented in [1] and is supported by a simplified 1D analytic description of fast wave propagation [2]. Using the Li-beam measurements during the separatrix position scans, the experimental $R_{c}$ versus $\left(R_{a n t}-R_{\text {Cutoff }}\right)$ was fitted using an exponential function of the form $R_{c} \propto R_{0} e^{-\beta k_{/ /}\left(R_{\text {ant }}-R_{\text {CuOff }}\right)}$. For each antenna the parameters $R_{0}$ and $\beta$ were determined from a fit of the $R_{c}$ measurements when changing the plasma outer position using the lowest $\left(5 \times 10^{21} \mathrm{el} / \mathrm{s}\right)$ gas rate from distant gas inlets.

Then, for each antenna the expected change in coupling resistance was estimated from the change in cut-off layer position $\Delta R_{\text {cutoff }}$ measured with the Li-beam diagnostic, when the gas level is increased from $5 \times 10^{21} \mathrm{el} / \mathrm{s}$ to $9.5 \times 10^{21} \mathrm{el} / \mathrm{s}$ as $\Delta R_{c} / R_{c_{-} \text {reference }}=\left(e^{\beta k_{/ /} \Delta R_{\text {Cuofff }}}-1\right)$. Consequently the expected coupling resistance increase rate $\kappa$ could be evaluated. The black rectangles in Figure 5 represent the range of coupling resistance increase rates aggregated for the 4 antennas, and expected from the measured shift in cut-off layer position. The uncertainties in density measurements are taken into account. In doing so, we have extended the definition of $\rho$ to describe the distance between the gas inlets and the location of density measurements. This procedure is equivalent to making an estimate of the coupling resistance improvement from the measured shift of the cut-off layer position, as if the antennas were located at the same location as the density measurement. From the good correspondence between measured and predicted $\kappa$ for $\rho \sim 1.5$ we can conclude that the improvement in coupling resistance for antennas located far from the gas inlet is consistent with the global density increase in the SOL. For $\rho \sim 0.8$ the SOL density measurements can only marginally explain the observed coupling improvement, suggesting that there are SOL modifications local to the antennas that are not measurable using the Li-beam diagnostic. There are two candidate explanations for this: 
- The gas inlet becomes magnetically connected to part of the antenna at a poloidal location that is different from the line-of-sight of the Li-beam;

- The increase in neutral gas close to the gas inlet plays a role in the improvement of ICRF coupling. The mid-plane neutral particle flux close to gas inlet A13 (measured by ionization gauge-15) is also shown in Figure 5. The neutral flux when injecting deuterium at $9.5 \times 10^{21}$ $\mathrm{el} / \mathrm{s}$ is plotted as a function of $\rho$ defined here as the distance between the inlets and the neutral flux measurement location. The neutral flux increases rapidly at locations close to the inlets. Neutral pressure in front of antenna could be an important parameter in the gas/RF interaction mechanism.

In summary, experiments have recently been carried-out on AUG in order to assess if local gas injection can improve ICRF antenna loading. The main outcomes are:

- The demonstration that, for midplane gas injection, local gas puffing can substantially increase antenna coupling without any adverse effects on ICRF system operation such as arcing at the antenna.

- The distance between the gas inlet and the ICRF antenna is an important parameter in the efficiency of the gas puff technique to improve antenna loading. As is illustrated in Figure 4 and Figure 5, injecting gas locally to the antenna maximizes the beneficial effect on antenna coupling and in turns could allow reduction the amount of gas to be injected and possible disturbances of plasma confinement. If this technique is to be used on ITER, we suggest that gas inlets are installed as close as possible to the ICRF antenna.

Whereas the effect of magnetic connection between the gas inlet and part of the antenna cannot be ruled out, there are several candidate mechanisms that could be responsible for the observed improvement in terms of local influence of ICRF waves and/or neutral particles:

a) Local SOL density increase from gas injection via neutral ionization by plasma electron impact.

b) RF/gas interaction resulting in plasma generation from enhanced neutral ionization in the far SOL in front of the antenna. In Ion Cyclotron Wall Conditioning experiments the collisional damping of the RF $\mathrm{E}_{/ /}$field on SOL electrons has been invoked to explain the generation of a plasma in front of ICRF antennas [11]. In the experiments reported here some pulses were run to assess whether the ICRF power has an effect on antenna coupling in the presence of local gas puffing. ICR-4 inlet was used in 
these pulses with injection rates of $5.2 \times 10^{21} \mathrm{el} / \mathrm{s}$ or $9.5 \times 10^{21} \mathrm{el} / \mathrm{s}$, a separatrix-antenna separation of $\sim 10.5 \mathrm{~cm}$, and with the ICRF power per antenna ranging between 0.2 MW and 0.8 MW. In this power range no noticeable change in the loading resistance was observed for Antenna-4. However, it is possible that a saturation in the plasma generation process was reached for the RF power range explored during the experiment (i.e. above $200 \mathrm{~kW}$ per antenna). By comparison, in AUG and JET ICRFWall Conditioning Experiments [12], plasmas with density in the order of few $10^{18} \mathrm{~m}^{-}$ ${ }^{3}$ were produced in front of the outer limiters by applying few hundred $\mathrm{kW}$ of RF power to $\mathrm{He} / \mathrm{D}_{2} / \mathrm{H}_{2}$ gas mixtures with neutral pressure in the order of $10^{-4} \mathrm{mbar}$.

c) Plasma/gas interaction resulting in a local modification of plasma flows in front of antennas. It is known that $\mathrm{E} \times \mathrm{B}$ flows are driven right in front of powered ICRF antennas, leading to local SOL density modifications [13].

d) Local modification of the transport in the SOL from gas injection, as was for example reported in JET when puffing $\mathrm{CD}_{4}$ gas in order to improve Lower Hybrid antenna coupling [14].

Given the mechanisms listed above, the enhanced antenna loading with gas puffing may not necessarily be interpreted as a one to one increase of RF power transmitted to the fast wave. But the technique remains a useful tool to improve ICRF system operation, provided the plasma facing components surrounding the antenna are not submitted to excessive heat-loads as a result. Local gas injection also proved to have a beneficial effect in reducing tungsten plasma content and local sputtering yield in AUG [15]. Further experiments are planned in the future to characterize the SOL plasma close to the gas inlet location and close to a powered antenna, using reciprocating probes, to address the question of the effect of RF power over a much wider power range and to further apply this technique to ICRF operation with H-mode plasmas. Finally, we note that this gas puffing technique, if needed for ICRF operation of next fusion devices, will need to be integrated into plasma scenario development, as gas fuelling can have an impact on plasma energy confinement [16].

Acknowledgments: The kind help and collaboration from IPP-Garching, from the AUG ICRF group, from the diagnosticians, and from the experiment leaders is warmly acknowledged. This work was carried out within the framework of the European Fusion Development Agreement (EFDA) under the task agreement WP09-HCD-02/04/UKAEA between EFDA and UKAEA (now CCFE) and WP09-HCD-02/04/IPP between EFDA and IPP. This work was funded partly by the RCUK Energy 
Programme under grant EP/I501045 and the European Communities under the contract of Association between EURATOM and CCFE. The views and opinions expressed herein do not necessarily reflect those of the European Commission.

\section{Appendix}

The calculation of antenna coupling resistance is detailed. The loading resistance $R_{L}$, of each transmission line feeding a strap can be calculated as:

$$
R_{L}=2 Z_{c}^{2} \frac{P_{n e t}}{V^{2}} \quad \text { (Equation A-1) }
$$

$\mathrm{Z}_{\mathrm{c}}$ is the transmission line characteristic impedance, $P_{n e t}$ is the net power in the transmission line toward the antenna, and $V$ is the maximum voltage (at antinodes) in the transmission line. $P_{n e t}$ is evaluated using directional couplers in the transmission line section located between the $3 \mathrm{~dB}$ Hybrid couplers and the double stub matching system (see Figure 2). It is assumed that the matching network is lossless and that power partition at the $\mathrm{T}$ junction feeding antenna straps is symmetrical. $V$ in each transmission line feeding a strap is evaluated via measurements of voltage probes along the line. The coupling resistance for each strap $R_{c}^{\text {strap }}$ is calculated subtracting the resistance associated to vacuum losses $R_{\text {vacuum }}^{\text {strap }}$ (typically $\sim 0.3 \mathrm{Ohms}$ ) to the strap loading resistance $R_{L}^{\text {strap }}$ :

$$
R_{c}^{\text {strap }}=R_{L}^{\text {strap }}-R_{\text {vacuum }}^{\text {strap }} \quad \text { (Equation A-2) }
$$

For each antenna, the coupling resistance is the average of the coupling resistance of each strap:

$$
R_{c}^{\text {antenna }}=\left(R_{c}^{\text {strap } 1}+R_{c}^{\text {strap } 2}\right) / 2 \quad \text { (Equation A-3) }
$$

\section{References}

1. V. Bobkov, et al., Nuclear Fusion 46 (2006) S469-S475

2. R. Bilato, M. Brambilla, D.A. Hartmann and A. Parisot, Nuclear Fusion 45 (2005) L5-L7

3. A. Messiaen, et al., Nuclear Fusion 50 (2010) 025026 (21pp)

4. M.-L. Mayoral, et al., 'On Maximizing the ICRF Antenna Loading for ITER plasmas', Proc. of the $23^{\text {rd }}$ IAEA Fusion Energy Conference 2010, Daejeon, Korea, ITR/P1-11

5. M.L. Mayoral et al., 17th Topical Conference on RF Power in Plasma, Clearwater, AIP conference proceedings 933, p55 (2007)

6. R.I. Pinsker, et al., $37^{\text {th }}$ EPS Conference on Plasma Physics Dublin (2010), (http://ocs.ciemat.es/EPS2010PAP/pdf/O4.124.pdf) 
7. L. Colas, et al., Plasma Phys. Control. Fusion 49 (2007) B35-B45

8. R Fischer, E Wolfrum, J Schweinzer, Plasma Phys. Control. Fusion, 50:085009, 2008

9. R. Fischer, E. Wolfrum, C. Fuchs, et al., Europhysics Conference Abstracts, Proc. of the 35th EPS Conference on Plasma Physics, Hersonissos, Crete Vol. 32D (2008), P-4.010

10. V. Lancellottia, D. Milanesio, R. Maggiora, G. Vecchi and V. Kyrytsya, Nuclear Fusion 46 (2006) S476-S499

11. A.I. Lysojvan, V.E. Moiseenko, O.M. Shvets, and K.N. Stepanov, Nuclear Fusion 321361 (1992)

12. A. Lyssoivan, et al., J. Nucl. Mater. (2011), doi:10.1016/j.jnucmat.2010.11.059

13. M. Becoulet, et al., Phys. Plasmas, Vol. 9, No. 6, June 2002.

14. G F Matthews, et al., Plasma Phys. Control. Fusion 44 689-699, (2002)

15. Vl. Bobkov, et al., Journal of Nuclear Materials 390-391 (2009) 900-903

16. G. Saibene, et al., Nuclear Fusion, Vol. 39, No. 9 (1999), pp 1133-1156 


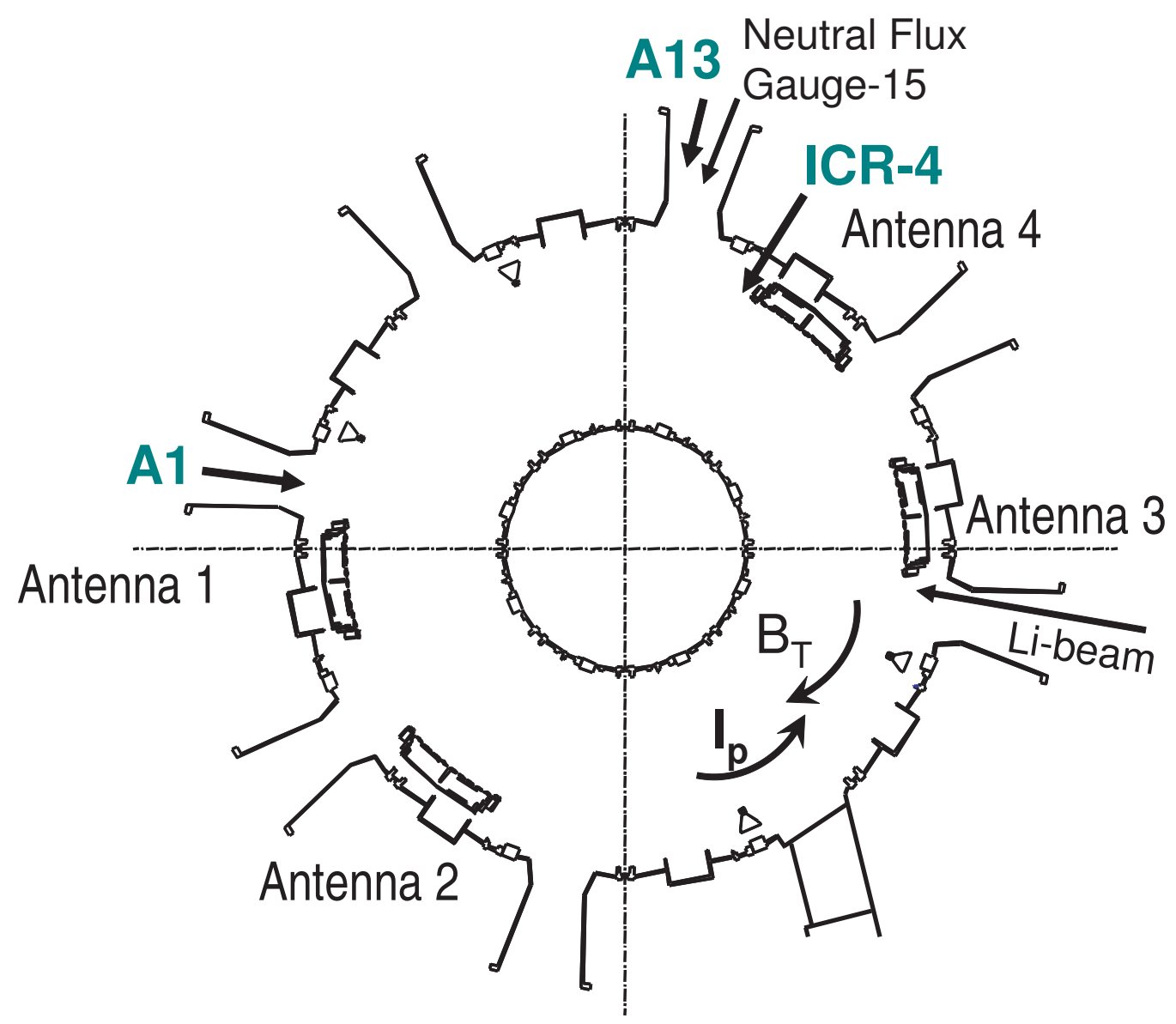

Figure 1. Top view of AUG showing the location of the ICRF antennas, the different gas inlets used during the experiment the Li-beam diagnostic and the neutral flux gauge-15. The chamber is pumped by cryopumps located in the lower divertor region. 


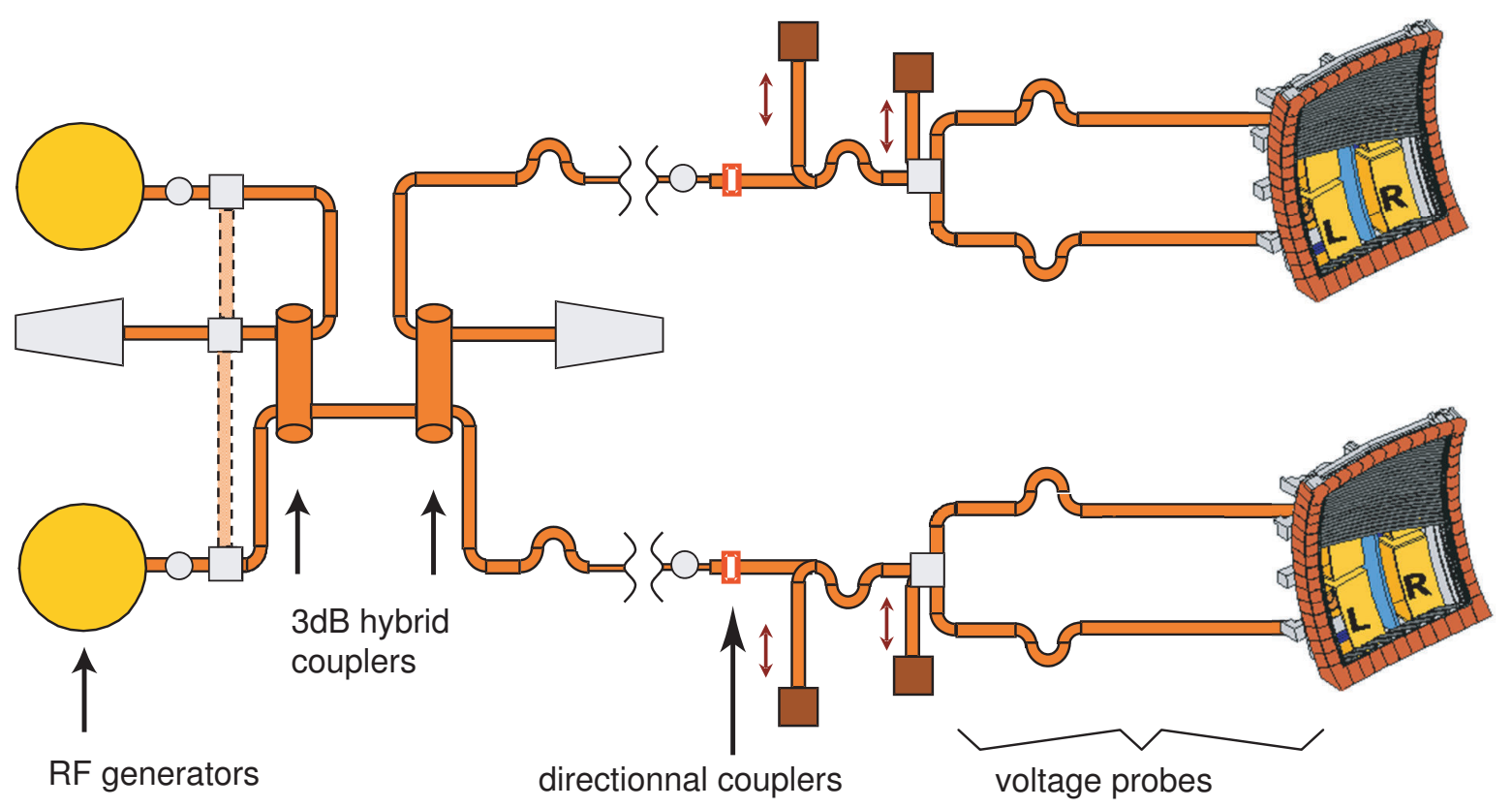

Figure 2. Schematic diagram representing the AUG ICRF system. Two antennas are paired through a $3 \mathrm{~dB}$ hybrid couplers arrangement. 


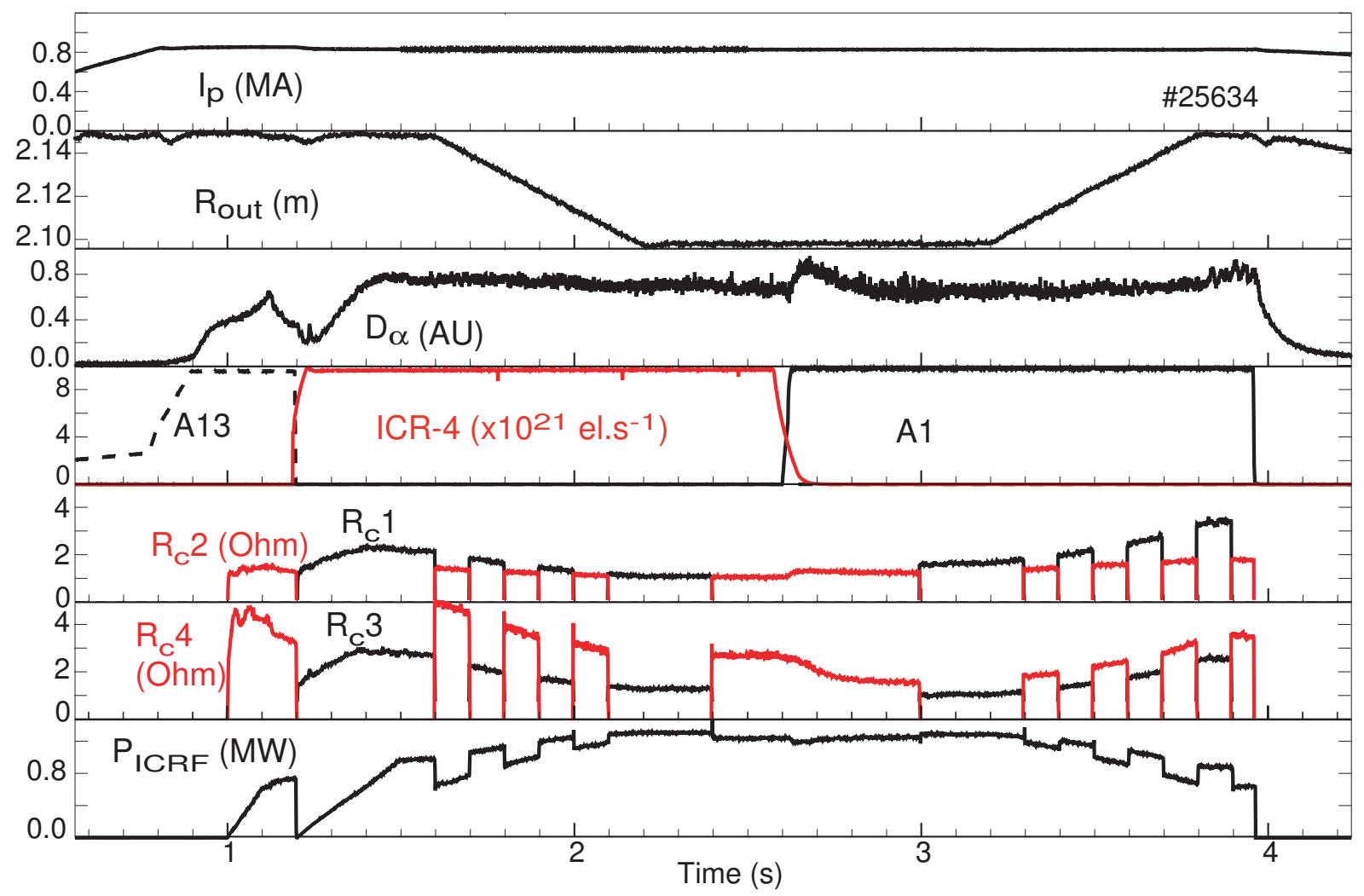

Figure 3. Time evolution of AUG pulse 25634, which is typical for this experiment. $I_{p}$ is the plasma current, $R_{\text {out }}$ is the outer position of the separatrix at the plasma midplane. $D_{\alpha}$ is the $D_{\alpha}$ emission in the outer region of the lower divertor, $\mathrm{A13} / \mathrm{ICR}-4 / \mathrm{A1}$ are the gas injection rates from the different inlets, $R_{c} 1 / R_{c} 2 / R_{c} 3 / R_{c} 4$ are the coupling resistance of antennas $1 / 2 / 3 / 4, P_{I C R F}$ is the total coupled ICRF power. 


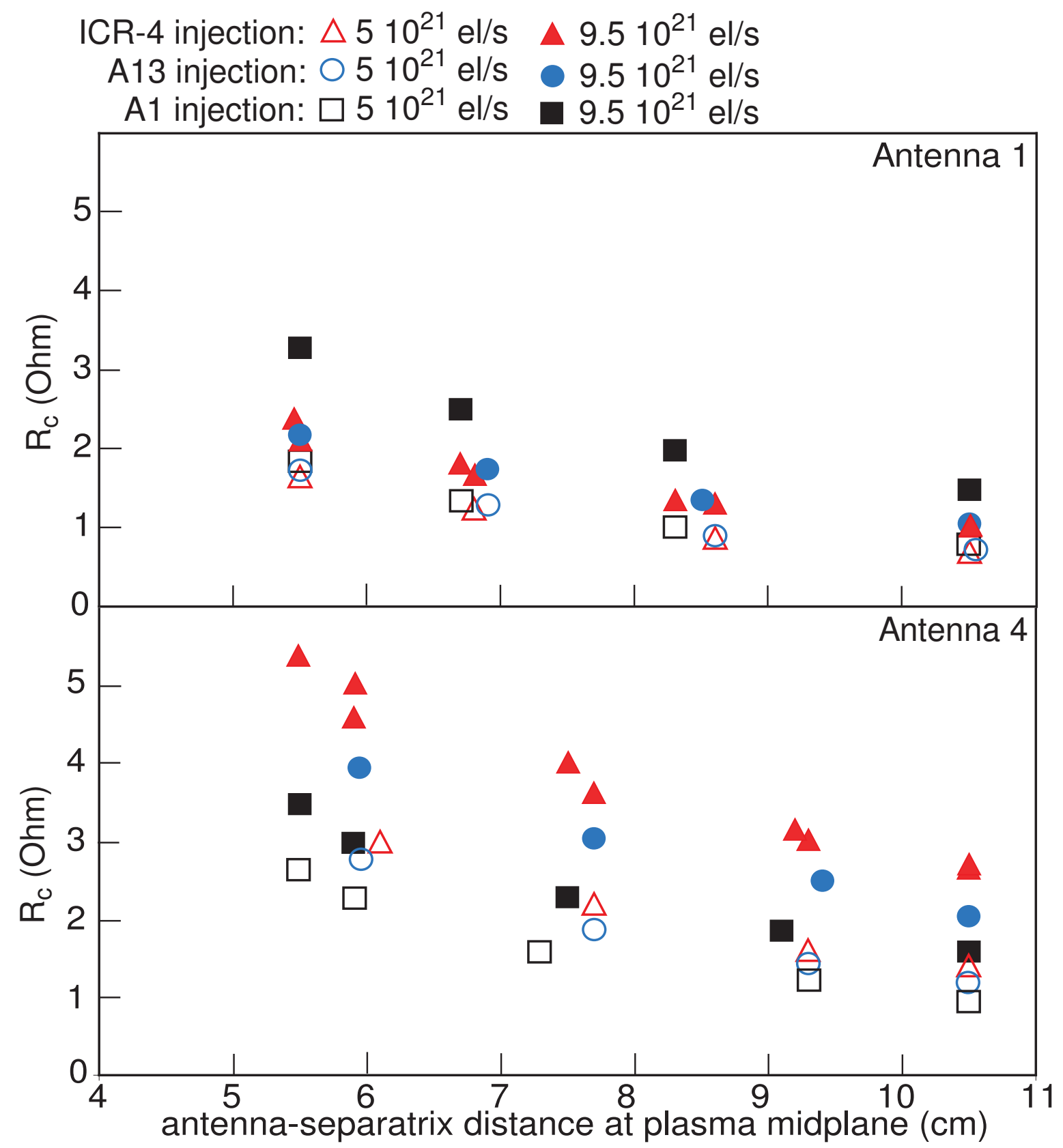

Figure 4. Coupling resistance of antennas 1 and 4 as a function of separatrix-antenna distance (measured from the antenna limiter) for 3 gas inlets (A1, A13, ICR-4) and two gas levels $\left(5 \times 10^{21}\right.$ el.s $^{-1}$ and $9.5 \times 10^{21}$ el. $\left.\mathbf{s}^{-1}\right)$. 


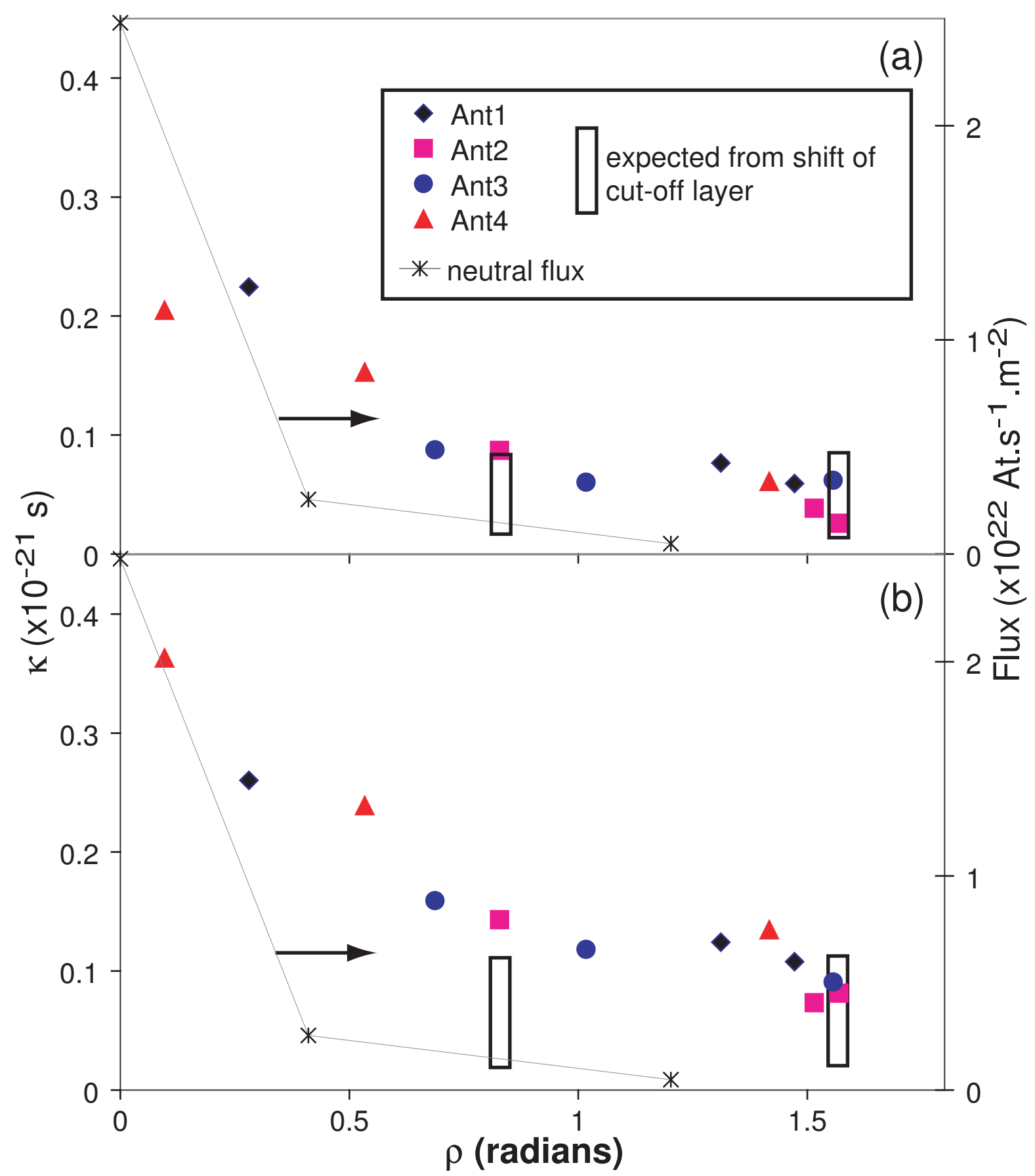

Figure 5. For all antennas the $R_{c}$ increase rate is plotted as a function of $\rho$ representing the distance between the antennas and the gas inlets for A1, A13 and ICR-4 (solid symbols). The separatrix-antenna distance is $5.5 \mathrm{~cm}$ on (a) and $10.5 \mathrm{~cm}$ on (b) (measured from the antenna limiter). The black rectangles represent the range of coupling improvement rate expected from the shift of the cut-off layer position when increasing the gas level. On the right $y$ axis the neutral flux measured by gauge-15 is also plotted as a function of $\rho$ (in this case $\rho$ represents the distance between gauge-15 and gas inlet). $10^{22}$ At. $^{-1} \cdot \mathrm{m}^{-2}$ corresponds to $\sim 6 \times 10^{-4}$ mbar assuming Maxwellian energy distribution function for the neutrals. 


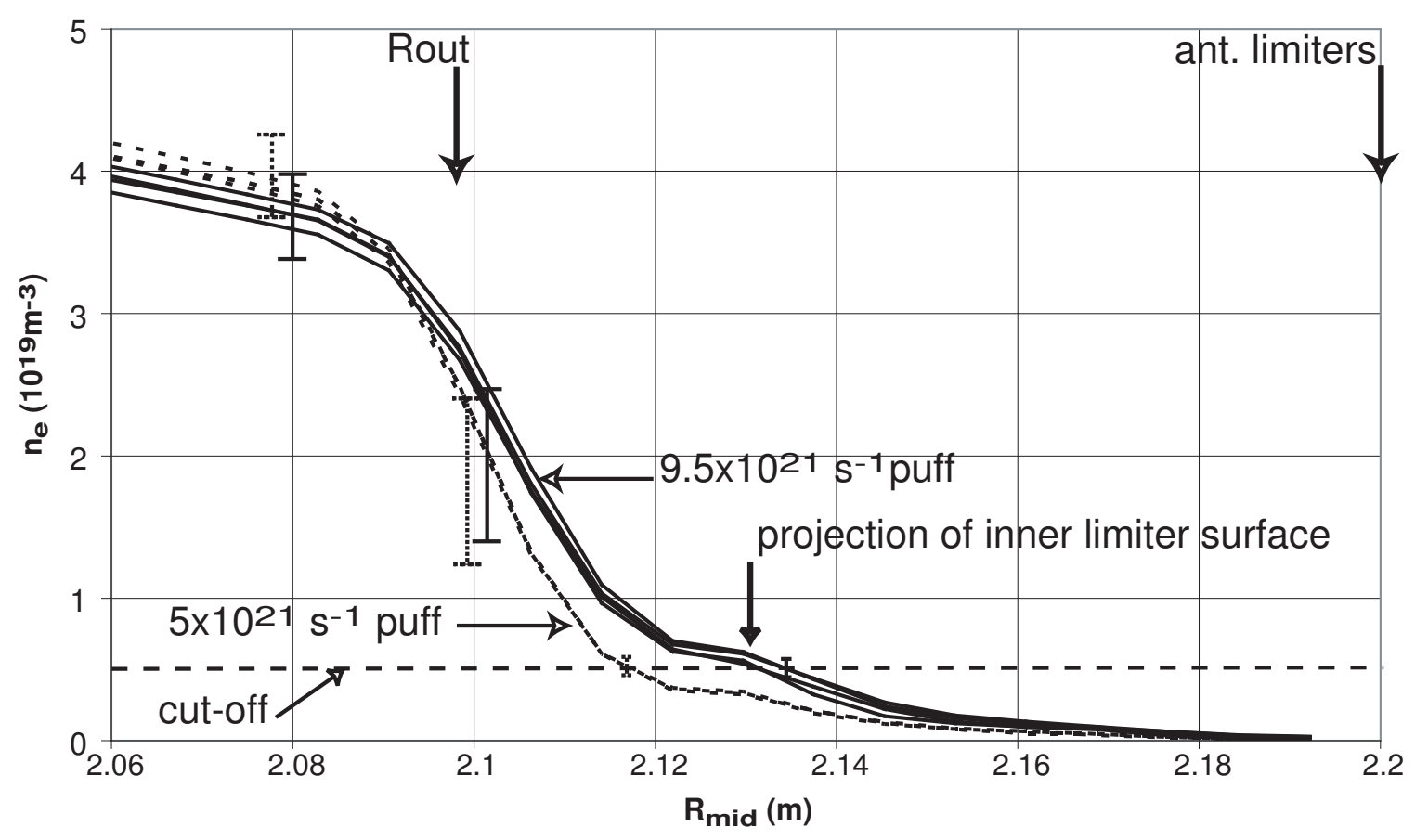

Figure 6. Electron density profiles (mapped onto the plasma mid-plane) for pulses 25633 and 25634.8 profiles are plotted, the dataset includes gas injection rate of $5 \times 10^{21} \mathrm{~s}^{-1}$ (dotted lines) and $9.5 \times 10^{21} \mathrm{~s}^{-1}$ from A1 and ICR-4 when antennas 1-3 or 2-4 are ON. Also indicated are the cut-off density $\left(\sim 5 \times 10^{18} \mathrm{~m}^{-3}\right)$, the outer-most plasma location from equilibrium reconstruction, the position of the projection of the inner limiter in the outer mid-plane, and the antenna limiter position at mid-plane. 